\title{
Factors Associated With Radiographic Vertical Bone Loss Around Implants Placed in a Clinical Study
}

\author{
Michael C. Manz* \\ * Previously, VA Medical Center, Ann Arbor, MI; currently, Department of Cariology, Restorative Sciences, and Endodontics, \\ University of Michigan School of Dentistry, Ann Arbor, MI.
}

The loss of vertical bone height over time has been assessed radiographically as part of the Dental Implant Clinical Research Group studies. Radiographs were assessed from implant placement, uncovering surgeries, and recall appointments. Overall, the study implants experienced most peri-implant vertical bone loss in the first year after placement, followed by a dramatic decrease in bone loss rate through the subsequent study intervals. Stratified analysis of data up to 72 months after implant uncovering indicates different bone loss patterns by: 1) arch; 2) jaw region; 3) case type; 4) bone quality; 5) surface type; 6) implant design; 7) smoking status; and 8) postoperative antibiotic treatment. These results will be used to build statistical mixed models to indicate which clinical factors are most predictive of peri-implant vertical bone loss, controlling for confounding and accounting for correlation of data over time and within study patients. Ann Periodontol 2000;5:137-151.

\section{KEY WORDS}

Bone loss/radiography; bone loss/prevention and control; dental implants.

This is U.S. Government-supported research, and there are no restrictions on its use. This paper has been approved for publication by the Dental Implant Clinical Research Group (DICRG), Department of Veterans Affairs. The views expressed in this article are those of the authors and do not reflect the views or policies of the U.S. Department of Veterans Affairs, nor imply endorsement by the U.S. Government.
$\mathrm{T}$ The need for controlled clinical studies to validly assess the safety and efficacy of the many different dental implant systems continues. Such studies can evaluate the relationships of patient and treatment variables to identify risk factors in providing such treatment, allowing for better treatment planning and improved patient care. Radiographic evaluation is one means of gaining information on tissue response in the peri-implant region, which can be used in selecting patients, restorative components, and procedures to maximize the probability of successful implant treatment.

Clinical studies of dental implants titled "The Influence of Implant Design, Application and Site on Clinical Performance and Crestal Bone" are presently being conducted by the Dental Implant Clinical Research Group at 32 Department of Veterans Affairs (DVA) medical centers and university research clinics. Changes in vertical bone height around placed implants are being evaluated radiographically as part of these studies to establish the expected pattern and extent of bone loss around implants in relation to different clinical and patient factors. Measurements are done on radiographs taken at implant placement, implant uncovering, prosthesis placement, 6 months after implant uncovering, 12 months after uncovering, and yearly thereafter. 
While establishing the extent, rate, and distribution patterns of vertical bone loss around endosseous implants, analysis of radiographic bone loss data is also being conducted to determine risk factors for bone loss around dental implants to aid in the decision-making process on whether and how to best provide dental implant treatment. While such information can be used to improve care provided to DVA patients, this information should also be generalizable and useful to all involved in providing dental implant treatment.

\section{BACKGROUND}

Osseointegration of implants has long been the advocated goal in dental implant treatment. Descriptions of bone reaction and osseointegration have been provided. ${ }^{1,2}$ Osseointegration depends on the relationship between biologic factors of bone and various clinical factors associated with dental implant treatment. This relationship has been addressed from different perspectives. Some authors have discussed how the response to dental implant treatment is dependent on patient characteristics and specific anatomical and physiological properties of the bone in which the implants are placed. ${ }^{3-7}$ Others have concentrated on the influence of implant characteristics such as implant coating and surface characteristics, implant design, and other clinical treatment factors such as the concomitant use of bone graft substitutes. ${ }^{8-11}$ Dental implant treatment has also been discussed as a specific means to preserve alveolar bone after tooth loss. ${ }^{12,13}$

\section{Peri-Implant Vertical Bone Loss}

Albrektsson et al. ${ }^{14}$ proposed criteria which are widely cited and used for the evaluation of dental implant success, including the criterion that vertical bone loss should be less than $0.2 \mathrm{~mm}$ annually following the first year of service of an implant. Criteria for dental implant success proposed in $1979^{15}$ included bone loss not greater than one-third of the vertical height of the implant. While these criteria provide evaluation guidelines, the definition of successful dental implant treatment should ultimately be based on the benefits of the treatment to the patient over the period of time that these benefits are derived, weighed against the negative aspects of the treatment, which would include subsequent treatment required for failing and failed implants. The extent of bone loss only indirectly affects the need for treatment or removal of dental implants through clinical parameters such as mobility. How much bone is required to sufficiently support a prosthesis remains a matter of debate.

New information on vertical bone loss around dental implants is being published continually from studies of various implant systems. Kapur ${ }^{16}$ followed bone loss in an earlier study of blade-type implants. Weber et al. ${ }^{17}$ found no statistically significant change in bone level between 1 and 2 years following placement of non-submerged implants. Baseline measurements were not made, but the authors estimated bone loss of about $0.6 \mathrm{~mm}$ around mandibular implants and $1.1 \mathrm{~mm}$ around maxillary implants in the first year following implant placement. Brånemark et al. ${ }^{18}$ reported bone loss of less than $0.1 \mathrm{~mm}$ annually for implants that were determined to be osseointegrated and that had been in function for 1 year prior to initiating the assessment. No information on the first year following implant placement was reported. Nishimura et al. ${ }^{19}$ likewise showed very small amounts of bone loss following prosthesis insertion. Malmqvist and Sennerby 20 reported a high proportion of implants, followed for up to 4 years, having vertical bone loss of more than $2 \mathrm{~mm}$, and many having bone loss greater than onethird the length of the implant. Arvidson et al. ${ }^{21}$ found minimal bone loss around mandibular implants in the 5 years after prosthesis insertion in fully edentulous cases. Lindquist et al. ${ }^{22}$ reported $0.5 \mathrm{~mm}$ of bone loss around implants in the first year following implant insertion, with a decreasing rate of bone loss during the 15 years of follow-up.

The relation of peri-implant bone loss to different implant and clinical factors has been investigated in many studies. Pilliar et al. ${ }^{23}$ reported differing vertical bone loss relative to different implant designs and surface characteristics. Karlsson et al. ${ }^{24}$ found differences in bone loss around implants following prosthesis insertion depending on the arch in which they were placed. Different periodontal parameters indicative of periodontal and gingival inflammation have been reported to be related to peri-implant attachment and bone loss. ${ }^{25-27}$ The influence of smoking and the combination of smoking with poor oral hygiene on peri-implant bone loss have been specifically investigated. ${ }^{28}$ Periimplant bone loss to the first thread of screw-type implants with conical and machined collar implant designs has been discussed. ${ }^{29,30}$ Surface characteristic modifications and treatments in the area of this collar have been proposed to deal with this problem. ${ }^{31,32}$

While earlier studies tended to assess and report bone loss following loading, some of the more recent studies have given attention to the period immediately following implant insertion. Bragger et al. ${ }^{33}$ reported median distances of bone from the implant shoulder and median changes over the first year after implant insertion. Median levels were between 2 and $3 \mathrm{~mm}$ below the shoulder, with median changes of slightly less than $1 \mathrm{~mm}$. Mean levels and changes would be expected to be somewhat greater than these median values. Pham et al. ${ }^{34}$ also found significantly higher bone loss rates in the period immediately following placement (with a rate of $1.28 \%$ of implant length per month) than in the post-loading intervals. They also 
found a difference in bone loss rate between the pressfit and screw-type implant designs used in the study. These varying results on vertical bone loss around dental implants and the relationship of bone loss to various clinical factors highlight the need for further studies in this area.

\section{Radiographic Measurement of Vertical Bone Loss}

To avoid further tissue trauma, vertical bone loss should be evaluated radiographically after implant uncovering. In radiographic measurement, the position of the coronal extent of bone-implant contact must be determined. To determine the total amount of vertical bone loss around dental implants, a baseline measurement must be made at the time of implant insertion. This measurement is made by assessing the distance from the level of bone to some point of reference on the implant. Subsequent measurements, using the same point of reference, can then be compared to baseline to estimate the amount of bone loss that has occurred subsequent to implant insertion.

Proportional measurements have been used in some large studies to speed the measurement process and address the problem of non-standardized radiographs. ${ }^{35-37}$ Studying bone loss around dental implants allows for easy adjustment of measurements on nonstandardized radiographs and determination of actual bone loss because the length of the dental implant is known. Direct measurements can also be easily converted to proportional measurements (e.g., the percentage of an implant in direct contact with bone) if desired. However, the variability and reliability of measurements with different radiographs and within and across examiners must be considered. ${ }^{38-40}$ Grondahl et al. $^{41}$ looked specifically at interobserver variation in the assessment of peri-implant bone levels. Other factors affecting the quality of information obtained from radiographic measurements include the radiographic equipment and techniques used, radiographic angulation, measurement technique, and the particular advantages and disadvantages associated with computer-based measurement, if such systems are used. ${ }^{42-53}$

\section{DICRG Dental Implant Studies}

The objectives of the DICRG studies are to assess the effects of implant design, application (partial/full denture, removable/fixed, multiple/single tooth), and location (maxillary/mandibular, anterior/posterior) on clinical performance and crestal bone. Patient and treatment factors will also be considered. Cases have been followed for up to 6 years after implant uncovering. These studies will assess implant survival along with specific complications and adverse responses associated with dental implant treatment. Other aspects of treatment will be monitored, including plaque and
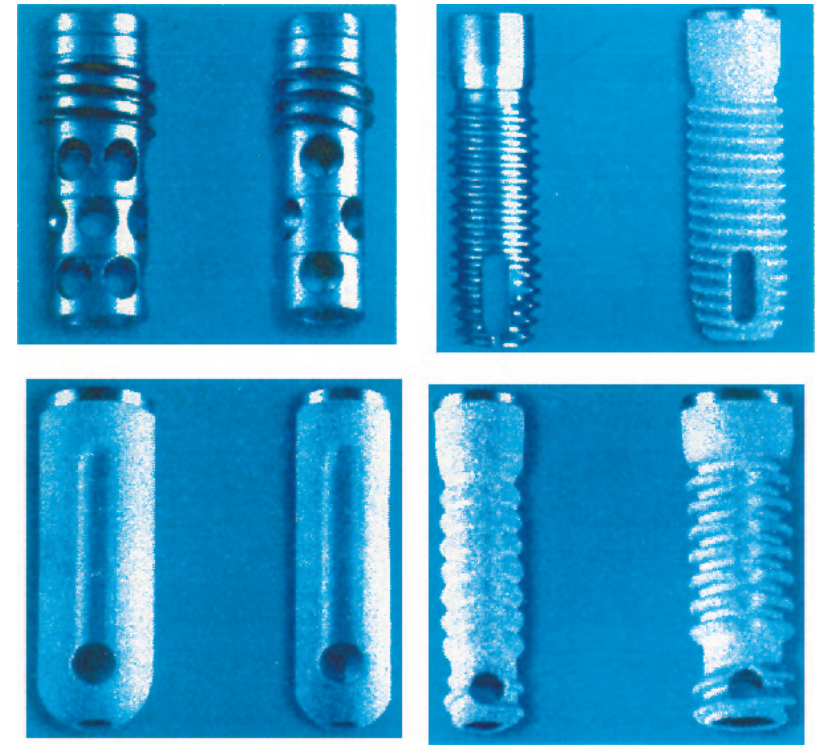

Figure I.

Implant designs used in the DICRG studies. Top left, Core-Vent (alloy basket); top right, Screw-Vent (HA, CP titanium, or titanium-alloy screw); bottom left, Bio-Vent (HA-coated cylinder); bottom right, Micro-Vent (HA-coated grooved cylinder).

calculus accumulation, soft tissue response, and patient satisfaction.

\section{MATERIALS AND METHODS}

\section{Study Population}

The DICRG studies are being conducted at 30 DVA medical centers and two university-based dental implant research clinics. Potential study subjects were recruited and initially screened for general health and oral conditions that could disqualify them from participation in the studies. Patients meeting study criteria and agreeing to participate were entered into the studies.

Study case types included mandibular and maxillary fully and partially edentulous cases, and maxillary anterior single-tooth replacement cases. Different implant designs ${ }^{\dagger}$ (Fig. 1) were randomly assigned to the sites for implantation in each study case, depending on the case type. The designated study surgeon at each study site determined the appropriate length and diameter for the implants to be placed. A designated clinician at each study site maintained, monitored, and collected data on the study implants over the course of the study. All study surgeons and clinicians met prior to the start of the study and annually during the study for planning and monitoring meetings that included study procedure standardization and calibration activities.

Sample sizes for the DICRG studies were calculated to ensure sufficient power to test the main hypotheses

$\dagger$ Spectra System, Core-Vent Corporation, DBA Paragon Company, Encino, CA. 
of the DICRG study. Final patient accrual goals for the studies were set using these calculations for minimum sample size requirements as a guide. More than one study case can be initiated and followed in a single study patient if conditions allow. The studies have accumulated a large number of study patients and cases, with totals of about 800 study patients, and approximately 1,000 study cases involving about 3,000 placed implants.

\section{Data Collection}

Radiographs were taken at each of the clinical procedure appointments including implant placement and uncovering surgeries, and final prosthesis insertion. Radiographs were then taken at follow-up appointments that were scheduled for all study patients based on the date of implant uncovering. These appointments were scheduled at 6 months, at 1 year, and then yearly thereafter for a period of 6 years.

Radiographs of the implant cases were sent to the project director's office where they were labeled, cataloged, and then measured by the author on a flat view box to the nearest $0.1 \mathrm{~mm}$ using vernier calipers. All measurements and analysis for this paper were completed by the author. The primary outcome of interest was bone level change over time. Therefore, systematic measurement error would not affect results. Avoidance of measurement drift over time was accomplished through periodic remeasurement of a sample of study radiographs to ensure agreement with previously completed measurements. With one investigator completing all measurements, interexaminer agreement was not an issue for this analysis. Computer analysis programs were evaluated and rejected for use in this study due to the shortcomings of using such programs for a multicenter study. A high level of standardization of radiographic techniques, angulation, radiographic unit settings, chemicals, etc., is virtually impossible to achieve and maintain over time at 32 sites spread across the country. Computerized analysis programs lose their advantages when radiographs are not highly standardized. Such aspects as contrast ranges indicating bone loss cannot be held constant with non-standardized radiographs. Blinded comparison and calibration activities were employed to ensure valid radiographic results in this study.

Vertical measurements of bone level adjacent to the implants were made from the top of the implant, which provides a fixed reference point. While two-stage implants generally should be inserted so that the top is flush with the level of the crestal bone, deviations often occur. Baseline measurements should be done so that these deviations can be accounted for in determining the change in bone level at subsequent appointments.

Calibration of the measured increments of bone change is necessary in determining actual bone loss from radiographic measurements, particularly on

\section{Radiographic Measurements}

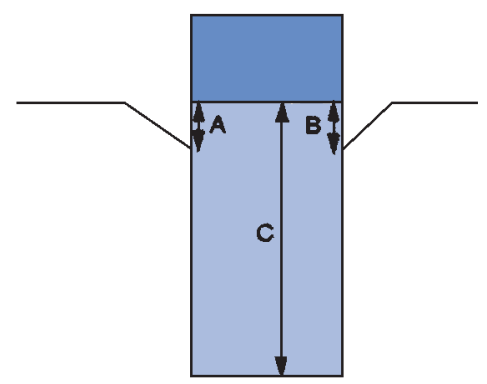

Measurement Average of Bone from Top of Implant $=(A+B) / 2$ Actual Average Distance $=(A+B)($ Known Length $) / 2 C$

Proportion of Implant Not Directly Apposed to Bone $=(A+B) / 2 C$

Figure 2.

Radiographic measurement sites and formulas used for analysis.

panoramic radiographs which generally provide an enlarged image of teeth and implants. Calibration is the process of correcting and standardizing measurements in radiographic analysis so that changes in bone over time may be determined accurately and validly. The measurement from the top of the implant to the point of the bone-implant interface is calibrated using the known and radiographically measured length of the implant. This calibration involves multiplying the vertical bone height measurements by the ratio of the known implant length to the measured implant length. Formulas used for calibration appear in Figure 2. These calibrated (i.e., actual) measurements from baseline and follow-up appointments were compared for a given implant to determine vertical bone height changes. Negative values were not assigned in cases where the bone level was above the level of the implant, because the extent of implant-bone contact was considered to be the factor of primary importance.

\section{Data Entry}

Measurements were entered onto paper forms that included case information generated by data management software, ${ }^{\ddagger}$ using databases with information entered from study forms sent to the DICRG Data Management Center (Ann Arbor, Michigan). Data were then entered into a data set using a data management and analysis package. $\S$ Entry was double checked for entry errors. Checks were run on the database to ensure clean, accurate data.

\section{Analysis of Data}

The raw data file created was converted into a data set for complete analysis. Variables were created and transformed as needed for analysis.

Valid analysis of the study data requires appropri-

\# Fox Pro, Microsoft Corporation, Redmond, WA.

$\S$ Epi Info., USD, Inc., Stone Mountain, GA.

\| SAS Institute Inc., Cary, NC. 
ate methods of data analysis. At the present stage of investigation, data collection is incomplete. Data have been collected on study implants for up to 72 months after implant uncovering. Due to limited availability of 72-month follow-up data, most results will be reported only up to 60 months. The results presented in this paper are primarily descriptive and intended for evaluation of trends seen in the data to this point. Results are presented on bone loss overall, and on bone loss stratified by various predictor variables. A more comprehensive analysis of the data will be conducted using the information from the present analysis and will include statistical modeling of the longitudinal data accounting for the correlated nature of the data within case and patient.

\section{RESULTS}

The mean actual bone levels calculated from the calibration formulas for the different study examination appointments are displayed in Table 1. Prosthesis insertion and 6-month follow-up examinations were usually completed within 2 months of each other, if not at the same appointment. At the 6-month follow-up, the average distance from the top of the implant to bone contact was $2.8 \mathrm{~mm}$. This distance increased one additional millimeter by the 72-month follow-up appointment, with very little change after the 36-month follow-up.

Bone level over time is viewed from a slightly different perspective in Figure 3. The average proportion of the implant that radiographically appeared to be in direct contact with bone at the different study evaluation points is displayed. The top portions of the bars indicate the mean proportion of implants not in direct

Table I.

Bone Level Relative to Top of Implant by Study Examination (in $\mathrm{mm}$ )

\begin{tabular}{l|c|c|c|c|c}
\hline Examination & $\mathrm{N}$ & Minimum & Maximum & Mean & $\mathrm{SD}$ \\
\hline Implant insertion & 2,111 & 0 & 5.6 & 0.6 & 0.6 \\
\hline Implant uncovering & 2,061 & 0 & 12.2 & 1.6 & 1.2 \\
\hline Prosthesis insertion & 1,376 & 0 & 8.9 & 2.7 & 1.2 \\
\hline 6-month follow-up & 1,762 & 0 & 9.9 & 2.8 & 1.2 \\
\hline I2-month follow-up & 1,937 & 0.2 & 11.4 & 3.1 & 1.3 \\
\hline 24-month follow-up & 1,804 & 0.5 & 11.1 & 3.5 & 1.3 \\
\hline 36-month follow-up & 1,481 & 1.0 & 10.9 & 3.6 & 1.3 \\
48-month follow-up & 1,037 & 0.9 & 10.5 & 3.8 & 1.4 \\
60-month follow-up & 630 & 0.9 & 10.2 & 3.8 & 1.5 \\
\hline 72-month follow-up & 128 & 1.3 & 8.3 & 3.7 & 1.2 \\
\hline
\end{tabular}

contact with bone, providing an indication of vertical bone loss relative to the length of the implant. The mean proportion, as evaluated radiographically, of the study implants not in direct contact with bone increased rapidly in the early stages and then stabilized with little additional support loss after the 24-month follow-up.

For those implants in which data were available for the beginning and end of study intervals, the average bone loss between study appointments is displayed in Table 2. This information is displayed graphically in Figure 4. Average bone loss between implant insertion and uncovering, and between implant uncovering and 6 months post-uncovering, was $1 \mathrm{~mm}$ and $1.2 \mathrm{~mm}$, respectively. Bone loss dropped to $0.4 \mathrm{~mm}$ for the 6 - to 12-month (a 6-month period) and 12- to 24-month (a 12-month period) intervals. Subsequent average annual bone loss up to the 60 -month evaluation was about $0.2 \mathrm{~mm}$ per year.

The remainder of the results primarily show the bivariate relationships of various predictor variables in the studies to radiographic bone loss. These results display the incremental bone loss occurring in the different study intervals, showing both the effect of the variables and the overall pattern of bone loss over time.

Figure 5 shows average vertical bone loss for implants placed in the maxilla compared with bone loss for implants placed in the mandible. There was more average vertical bone loss around implants placed in the maxilla than around those placed in the mandible in every study interval, with larger relative differences in later study intervals. There was also more bone loss for implants placed in anterior regions of the maxilla and mandible than in posterior regions

\section{Direct Implant to Bone Apposition as a Proportion of Total Implant Surface}

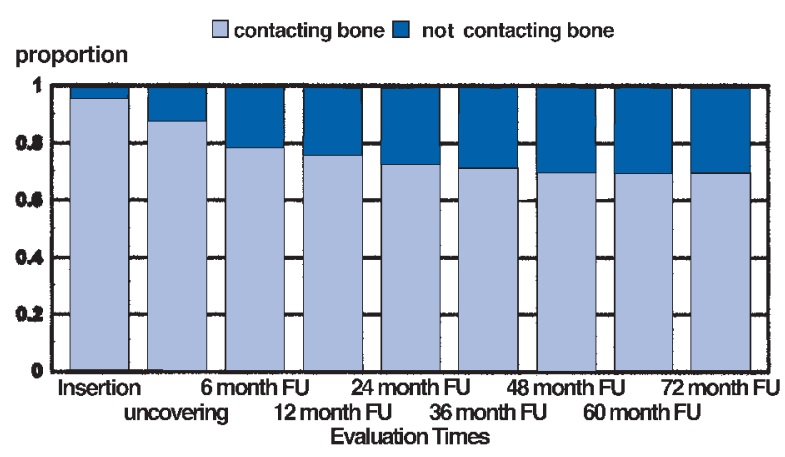

Figure 3.

Direct implant-to-bone apposition as a proportion of total implant surface. 
Table 2.

Bone Level Change Between Study Examinations (in $\mathrm{mm}$ )

\begin{tabular}{lccccc}
\hline \multicolumn{1}{c}{ Examination } & $\mathrm{N}$ & Minimum & Maximum & Mean & $\mathrm{SD}$ \\
\hline Stage I to Stage 2 & 1,727 & -1.3 & 11.8 & 1.0 & 1.0 \\
Stage 2 to 6 months & 1,482 & -1.6 & 8.4 & 1.2 & 1.1 \\
6 to 12 months & 1,405 & -2.7 & 7.0 & 0.4 & 0.7 \\
12 to 24 months & 1,441 & -2.3 & 7.1 & 0.4 & 0.9 \\
24 to 36 months & 1,288 & -2.7 & 4.9 & 0.2 & 0.8 \\
36 to 48 months & 914 & -2.4 & 5.5 & 0.2 & 0.8 \\
48 to 60 months & 511 & -2.7 & 4.4 & 0.2 & 0.7 \\
\hline
\end{tabular}

\section{Mean Vertical Bone Change During Study Evaluation Intervals}

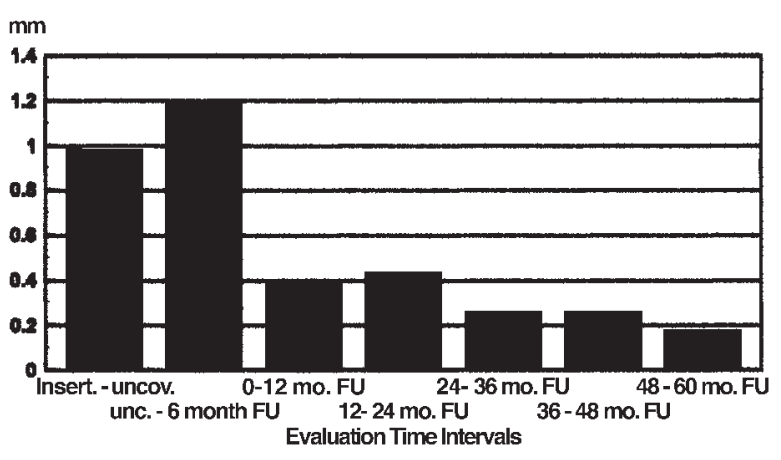

Figure 4.

Mean peri-implant vertical bone change for study intervals.

\section{Vertical Bone Change in Study Intervals by Anterior/Posterior Position}

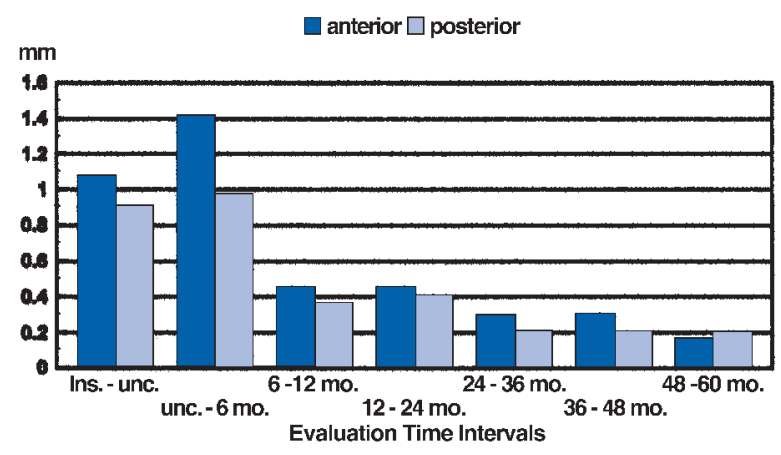

Figure 6.

Mean peri-implant vertical bone change for study intervals: anterior versus posterior. generally through the study intervals (Fig. 6). In the case of anterior versus posterior positioning, however, the larger relative differences were in the early study intervals.

These findings are consistent with the results displayed in Figure 7, which shows a further breakdown of jaw location. The tendencies for more bone loss in the maxilla than the mandible and more bone loss in anterior regions than posterior regions are still apparent. The chart shows the anterior versus posterior differences dominating early intervals and maxillary versus mandibular differences more apparent in later study intervals.

Vertical bone loss by bone quality (as evaluated at the implant placement surgery) is shown in Figure 8 . The figure shows a slight tendency for more vertical bone loss with increasing numeric bone qual-

\section{Vertical Bone Change in Study Intervals by Arch}

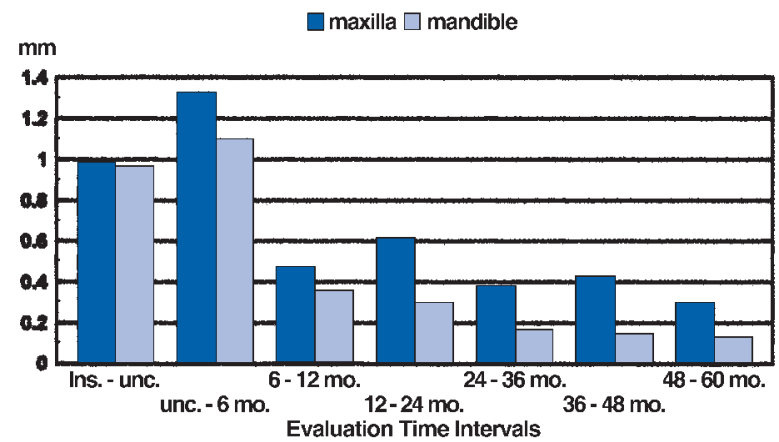

Figure 5.

Mean peri-implant vertical bone change for study intervals: maxilla versus mandible.

\section{Vertical Bone Change in Study Intervals by Jaw Location}

$\square$ Maxillary Anterior $\square$ Maxillary Posterior $\square$ Mandibular Anterior $\square$ Mandibular Posterlor $\mathrm{mm}$

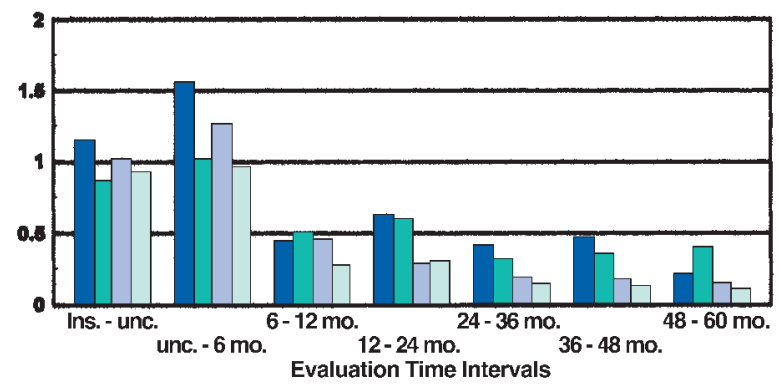

Figure 7.

Mean peri-implant vertical bone change for study intervals by arch location. 
ity-type designations (generally corresponding to less dense bone), particularly between the implant uncovering and 6-month follow-up examination.

Figure 9 displays mean vertical bone loss in study intervals by case type. In early study intervals, more bone loss was seen in completely edentulous and maxillary single-tooth cases, with less bone loss in partially edentulous cases. In later study intervals, all maxillary case types showed more bone loss than mandibular case types, with a continued indication of less bone loss in partially edentulous cases than completely edentulous and single-tooth cases.

Vertical bone change for HA-coated versus non-HA implants is compared for the study intervals in Figure 10. Non-HA-coated implants showed more vertical bone loss in the implant insertion to implant uncovering and uncovering to 6-month follow-up intervals.

\section{Vertical Bone Change in Study Intervals by Bone Quality}

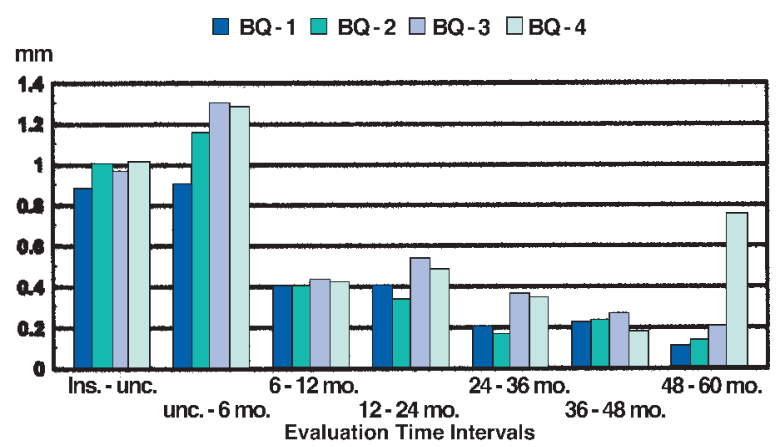

Figure 8.

Mean peri-implant vertical bone change for study intervals by bone quality score.

\section{Vertical Bone Change in Study Intervals by HA vs non-HA}

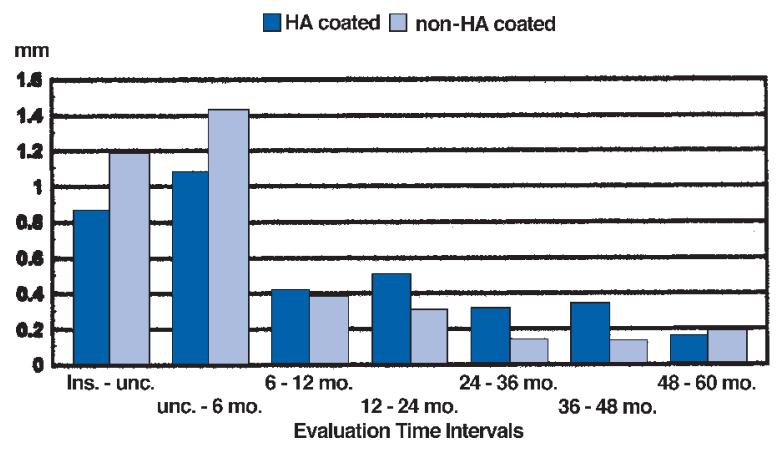

Figure 10.

Mean peri-implant vertical bone change for study intervals: HA versus non-HA.
Subsequent intervals showed a reversal, with HAcoated implants consistently showing greater bone loss.

Figure 11 displays the comparison of bone loss between implants with and without HA coating stratified by arch. The figure shows a trend of more bone loss in non-coated implants and implants placed in the maxilla up to 6 months after uncovering. Figure 12 displays a further breakdown of data by HA, arch, and anterior/posterior location.

Figure 13 shows bone change for the different specific implant designs used in the study. In the early study stages, the non-coated metal implants showed the greatest amount of bone loss, particularly the commercially pure titanium screw. All implants showed similar amounts of bone loss in the 6- to 12 -month

\section{Vertical Bone Change In Study Intervals by Case Type}

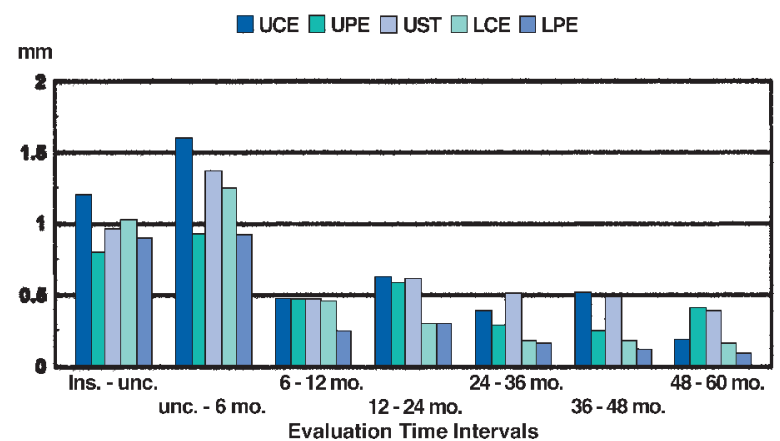

Figure 9.

Mean peri-implant vertical bone change for study intervals by case type. UCE = upper completely edentulous; UPE = upper partially edentulous; UST = upper single tooth; $L C E=$ lower completely edentulous; $L P E=$ lower partially edentulous.

\section{Vertical Bone Change in Study Intervals by Arch and HAnon-HA}

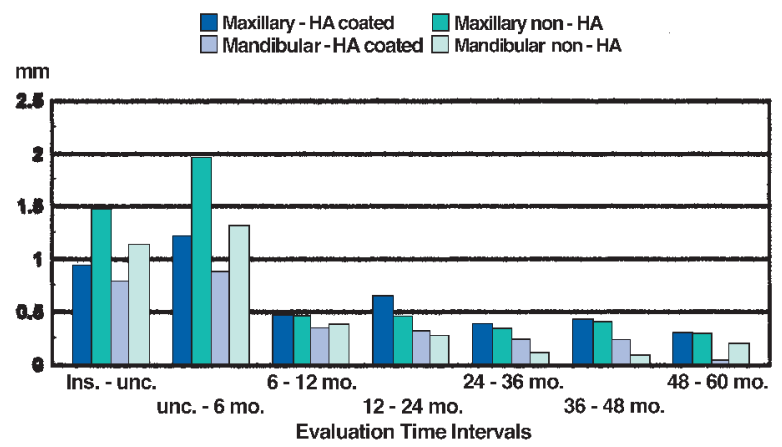

Figure I I.

Mean peri-implant vertical bone change for study intervals: HA versus non-HA and maxilla versus mandible. 
follow-up interval. Subsequently, the non-coated implants appeared to have less bone loss than the HAcoated screw and grooved cylinder implants, with the alloy basket showing the least amount of bone loss, followed by the alloy screw and the commercially pure titanium screw. The HA-coated cylinder showed less bone loss through the study intervals than the other HA-coated designs and the commercially pure titanium screw.

Bivariate relations of patient-specific factors to bone loss were also evaluated. Figure 14 shows bone loss by gender. Females had less bone loss in the insertion to uncovering interval, but then had more bone loss in subsequent intervals up to the 36-month follow-up. It should be noted that study subjects were primarily derived from DVA dental clinic patients. There

\section{Vertical Bone Change in Study Intervals by $\mathrm{HA}$ and Arch Location}

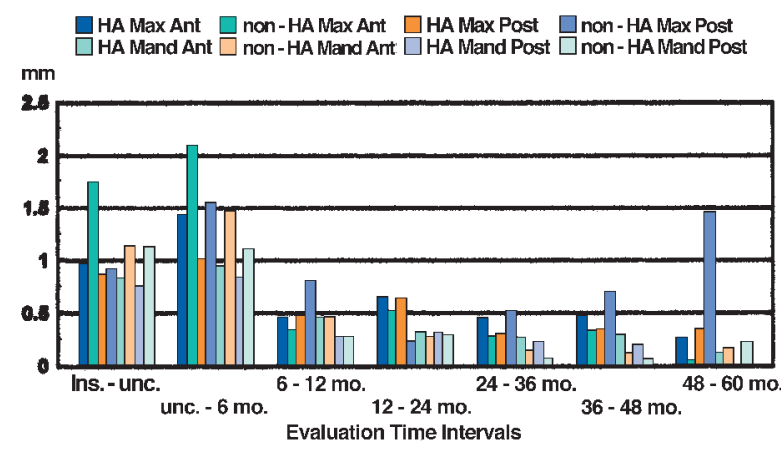

Figure $/ 2$.

Mean peri-implant vertical bone change for study intervals: HA versus non-HA and arch location.

\section{Vertical Bone Change in Study Intervals by Gender}

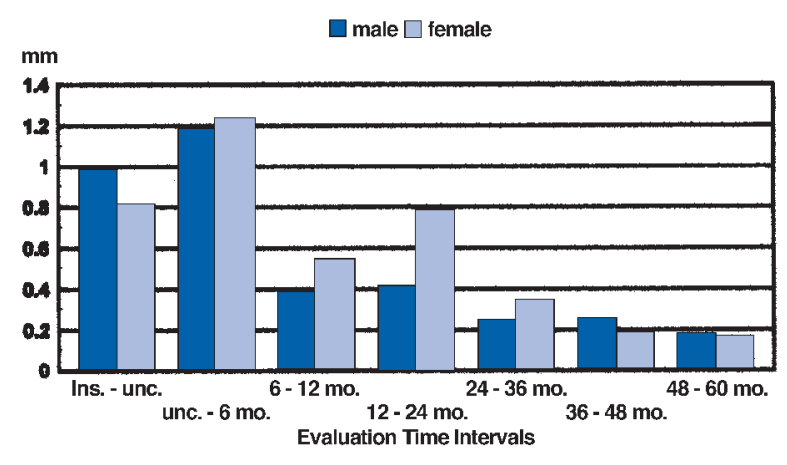

Figure 14.

Mean peri-implant vertical bone change for study intervals by gender. were relatively few female patients included in this study, with around 70 females represented in early study intervals, dropping to 12 females in the 48- to 60-month interval for which data had been collected.

Figure 15 shows bone loss stratified by race/ethnicity. There was a very slight pattern of more bone loss in early study intervals up to the 12-month followup in whites than in African-Americans. Subsequent intervals showed slightly less bone loss in whites than in African-Americans. No clear pattern was apparent for the "other" races combined category. Again, the numbers in the "other" category were relatively small.

The relation of ASA medical status ${ }^{54}$ to bone loss is shown in Figure 16. Bone loss appeared inversely related to ASA medical status code up to the 6-month follow-up. After the 6-month follow-up, the trend turned

\section{Vertical Bone Change in Study Intervals by Implant Design}

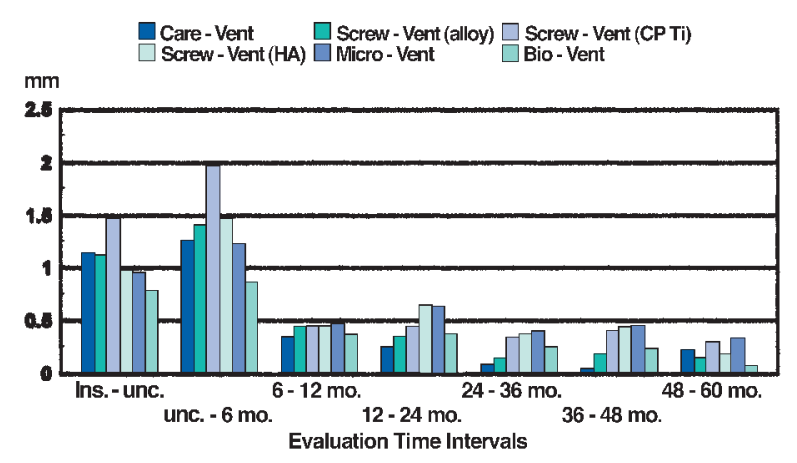

Figure 13.

Mean peri-implant vertical bone change for study intervals by implant design.

\section{Vertical Bone Change in Study Intervals by Race/Ethnicity}

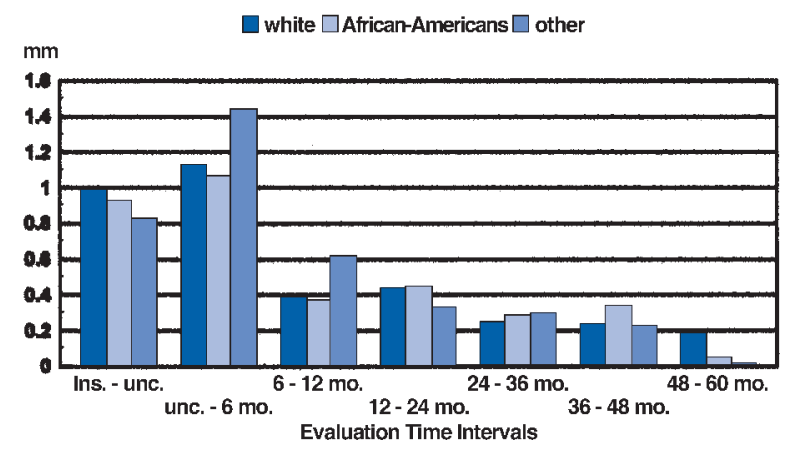

Figure 15.

Mean peri-implant vertical bone change for study intervals by race/ethnicity. 
to a direct relationship; patients with more severe systemic health problems appeared to have more bone loss around placed implants. The differences between categories 1 and 2 were small. Relatively few patients with ASA status of 3 were included in the study.

Figure 17 displays the relationship of smoking to peri-implant bone loss. Although not all study intervals showed the same trend, current smokers generally had more bone loss than non-smokers and former smokers. Little difference in bone loss was apparent between former smokers and those with no history of smoking.

Surgeons placing the study implants were dichotomized based on the number of implants they had placed prior to the study. Surgeons placing 50 or more

\section{Vertical Bone Change in Study Intervals by ASA Medical Status}

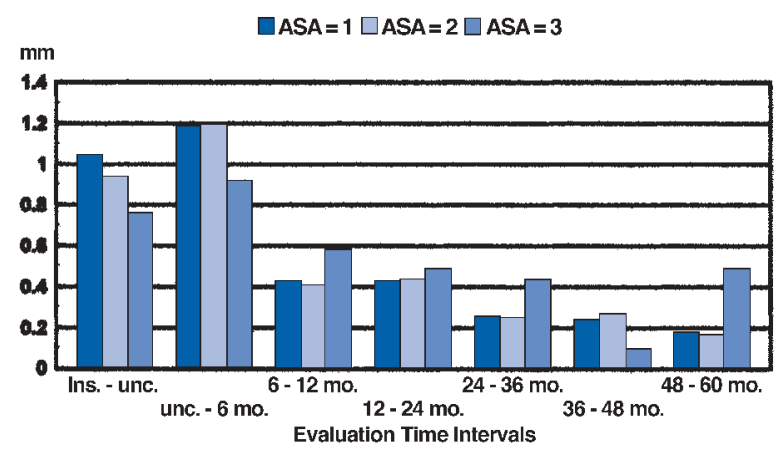

Mean peri-implant vertical bone change for study intervals by ASA medical status.
Figure I6.

implants prior to the study were classified as "more experienced," while surgeons placing less than 50 implants prior to the study were placed in the "less experienced" category. This cut-point results in nearly equal numbers of study surgeons in the "more" and "less" experienced categories for purposes of analysis. This analysis procedure is described in a previous paper. ${ }^{55}$ Figure 18 shows little relationship of bone loss to this prior experience categorization.

Figures 19 and 20 display bone loss in relation to the use of antibiotic treatment in conjunction with implant placement surgery. There does not appear to be any clear pattern between the use of preoperative antibiotics and bone loss (Fig. 19). Patients treated with postoperative antibiotics experienced slightly more

\section{Vertical Bone Change in Study Intervals by Smoklng Status}

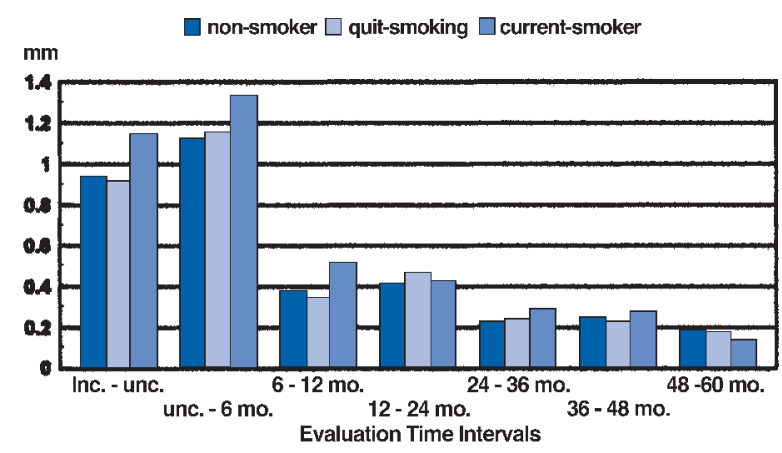

Figure 17.

Mean peri-implant vertical bone change for study intervals by smoking status.

\section{Vertical Bone Change in Study Intervals by Preoperative Antibiotics}

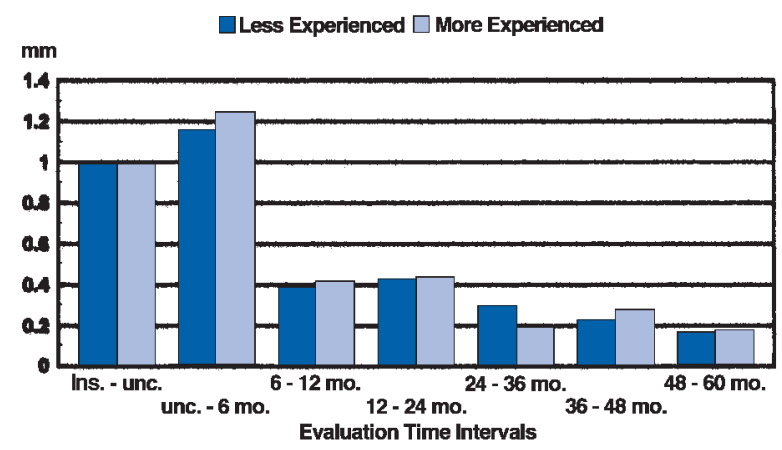

Figure 18.

Mean peri-implant vertical bone change for study intervals by surgeon experience.

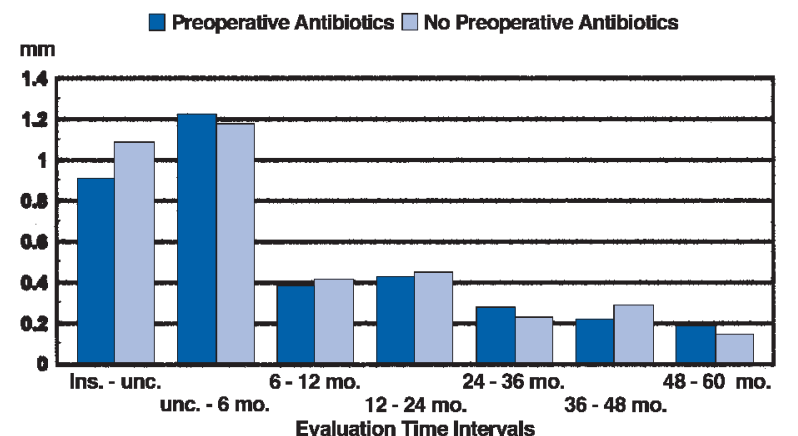

Figure 19.

Mean peri-implant vertical bone change for study intervals by use of preoperative antibiotics. 


\section{Vertical Bone Change in Study Intervals by Postoperative Antiblotics}

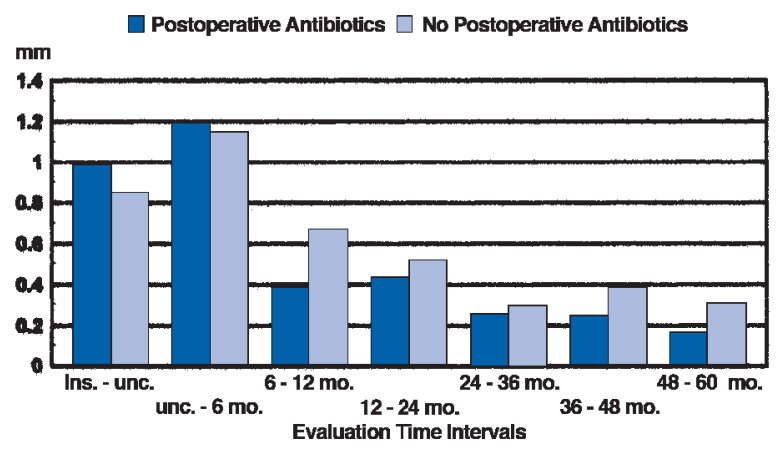

Figure 20.

Mean peri-implant vertical bone change for study intervals by use of postoperative antibiotics.

bone loss up to the 6-month follow-up, but appeared to experience less bone loss in all subsequent study intervals (Fig. 20).

\section{DISCUSSION}

The actual mean implant-bone contact positions relative to the top of study implants over the study are displayed in Table 1 . The values displayed are the calibrated means using the known implant length to measured implant length to adjust the measurements from the top of the implant to the radiographically visualized point of implant-bone contact. The data show a relatively rapid recession of this contact point between implant insertion and the 6-month followup appointment, followed by a marked stabilizing of this implant-bone contact level. Between implant insertion and the 6-month post-uncovering follow-up (representing a total period of about 1 year), the mean distance of bone contact receded from $0.6 \mathrm{~mm}$ to $2.8 \mathrm{~mm}$ below the top of the implant. In the subsequent 66 months, the mean level dropped only an additional millimeter.

Figure 3 displays implant-bone contact level as a proportion of implant length. The upper sections of the bars indicate the average proportion of the study implants that do not appear radiographically to be in direct approximation to bone, providing an indication of vertical bone loss relative to implant length in the study evaluations. On average, about $95 \%$ of the study implants appeared radiographically to be in direct contact with bone at implant insertion. This proportion dropped to $87 \%$ at implant uncovering and $78 \%$ at 6 months post-uncovering. By the 72-month follow-up, the percentage of vertical implant-bone contact dropped to about $69 \%$. But again, this percentage of vertical implant-bone contact became fairly stable in later study intervals, dropping only about 3\% between the 24- and 72-month follow-ups, or less than $1 \%$ per year. This information can be evaluated and related to present ideas on the percentage of direct bone-implant contact necessary to bear the mechanical stress and loading forces placed on dental implants when prosthetic restoration has been completed.

The prominent theme in the data over all implants is the relatively rapid loss of vertical bone immediately after implant insertion, followed by dramatically decreased amounts of bone change. While the bone support for study implants stabilizes significantly, the continuing minimal bone loss around implants must be evaluated. The remainder of the results address the incremental changes during the study intervals over time, which emphasizes this decrease in bone change over time. It also allows for differences in the smaller amounts of change during later intervals to be seen more clearly so that different factors can be evaluated for effects that may change over time. Results are provided for study interval bone changes overall and by various and sometimes overlapping relationships of different patient, procedure, and implant variables. Results for bone change during study intervals are reported only to the 60 -month follow-up because data are available for too small a number of implants in the 60- to 72-month interval to provide reliable results.

It is also important to note that when evaluating means for bone loss around study implants, the distribution of bone loss measurements must be considered. Bone change measurements indicating bone gain over time are uncommon. The distributions of measurements are centered around small amounts of bone loss and skewed toward higher measurements of bone loss. The median bone loss across implants is less in this situation than the means, with greater bone loss measurements pulling up the means. The means therefore partially reflect the effect of small numbers of implants with large amounts of bone loss. Measurement distributions are less skewed in later study intervals, but still remain somewhat skewed toward high bone loss levels. While not specifically addressed in this paper, additional research could focus on factors associated with implants showing rapid and extensive loss of vertical bone height.

Figure 4 displays the bone change over all DICRG implants in the study intervals up to 60 months. The average study implant experienced about $1 \mathrm{~mm}$ bone loss between implant insertion and uncovering, and another $1.2 \mathrm{~mm}$ of bone loss between implant uncovering and 6 months post-uncovering. The mean bone loss in each of the 6- to 12- and 12- to 24-month intervals was about $0.4 \mathrm{~mm}$. In each 1 -year interval from the 24- to 60-month follow-up, the mean bone loss was about $0.2 \mathrm{~mm}$. It is difficult to compare bone 
loss in the early stages of the DICRG studies to other studies because publications from other studies, while mentioning more bone loss immediately after implant insertion, have generally only provided data for periods after implant loading. The results from the DICRG studies do show that after the initial stages, the rate of vertical bone loss around DICRG study implants declines substantially and levels off at about $0.2 \mathrm{~mm}$ per year.

More vertical bone loss appears to be associated with implants placed in the maxilla than the mandible, and with implants placed in anterior regions than in posterior regions (Figs. 5 and 6). There was a slight difference in trend over the study intervals for these two variables, however. Differences by arch were apparent over all the study intervals, but appeared more marked in later study intervals. Differences by anterior/posterior position, on the other hand, showed more dramatic differences in the intervals up to the 6-month follow-up, followed by less dramatic differences. This difference in trends is further supported by Figure 7, which shows bone change results stratified by arch and anterior/posterior location. In the earlier study intervals, the differences between anterior and posterior implants dominated these further stratified results. The maxillary mandibular differences predominated the results for later study intervals.

Bone quality scores may interrelate with implant placement location variables in these studies. Bone quality has been stated to be associated with arch and anterior/posterior location in the arch. ${ }^{56}$ The differing distributions of bone quality by arch and anterior/posterior location for the DICRG study have been reported previously. ${ }^{57}$ Vertical bone loss by bone quality scores is shown in Figure 8. There was a slight trend for more bone loss around implants placed in less dense bone, particularly in the early study intervals. Differences by bone quality were not very dramatic, but possible confounding relationships with implant location and other variables should be investigated. Note that data were available for only 12 implants placed in type 4 bone for the 48- to 60-month interval, which provide an unreliable bone change estimate.

Case type is also associated with the previously mentioned variables. Implants for completely edentulous cases were placed in anterior regions of the jaws, as were the maxillary single-tooth replacement cases. Partially edentulous study cases involved implants placed only in the posterior regions of the jaws. Vertical bone change by case type is shown in Figure 9. These results paralleled the results seen for implant jaw location. In the early study intervals, the largest differences were seen between the partially edentulous (posteriorly placed cases) and the other anteriorly placed cases. The largest differences in the later study intervals were between maxillary and mandibular case types. There also may have been a tendency for more bone loss around implants in completely edentulous cases, particularly in the early study intervals. Patient periodontal factors that may have contributed to tooth loss may also have affected the bone support around study implants.

More bone loss was associated with non-HA implants than HA-coated implants in the early study intervals (Fig. 10). But interestingly, this trend reversed after the 6-month follow-up. All subsequent intervals showed more average bone loss for HA-coated implants than the non-HA implants. It is possible that these findings may relate to the immediate biologic reaction to the implant surface at implant placement. Bone may initially react more favorably to the HA coating, but changes in the HA or periodontal factors over time may negate this early advantage. The results indicate that after 24 months post-uncovering, the HA implants experienced more total bone loss than the non-HA implants, although the differences were small.

Figure 11 provides additional interesting results for HA-coated versus non-coated implants stratified by arch. The same basic trends described for the bivariate relationships of arch to bone loss and HA coating to bone loss are still apparent in this figure. In the early study intervals, less bone loss was associated with HAcoated implants and implants placed in the mandible. Later study interval results showed a continued greater bone loss in the maxilla and the reversal to greater bone loss with HA-coated implants. There does not appear to be much difference by HA/non-HA in maxillary implants during these later study intervals, however.

Further stratification by anterior/posterior arch location (Fig. 12) shows that this lack of difference for HA versus non-HA in the maxilla is accounted for by the posterior maxillary implants. Most of the general trends can still be seen in Figure 12, including the early differences by anterior/posterior location and the later obvious differences by arch. Generally, the HA-coated implants showed the same trend from less bone loss in early study intervals to more bone loss in later study intervals. However, in the maxillary posterior region, HA-coated implants lost less bone than non-HA implants in all but the 12- to 24-month interval. Few implants were in this group; data were available for only 8 maxillary posterior non-HA implants in the 48to 64-month interval.

Study interval bone loss by implant design is shown in Figure 13. The implants have been arranged with the three non-HA implant designs on the left side of the bar groupings and the three HA-coated implant designs on the right side of the groupings. The general trend for HA versus non-HA implants seen previously is still apparent. Early intervals 
showed more bone loss in the non-HA implants. Again, at 6 months post-uncovering, HA-coated implants generally began to show more bone loss than the non-HA implant designs. There were some deviations from this general pattern, however. The commercially pure titanium screw consistently had more bone loss than the other non-HA designs, and continued to show more bone loss than the HA-coated cylinder. The cylinder design consistently showed less bone loss than the other HA-coated designs, with the least bone loss of all designs up to the 6-month follow-up. After 6 months, the HA-coated cylinder design experienced similar bone loss to the alloy screw. The most dramatic decrease in bone loss over the study intervals occurred with the alloy basket design. There seems to be an indication that the three screw designs (HA, CP titanium, and titanium-alloy) experience greater bone loss than the other designs.

The interrelationships between the different study variables must also be considered when evaluating the results for bone loss by implant design in Figure 13. The different implant designs were not used in all locations or applications in the DICRG studies. Alloy basket implants were placed only in mandibular cases. HA-coated grooved cylinder implants were placed only in the maxillary single-tooth and partially edentulous cases. Titanium-alloy screw implants were placed only in mandibular fully edentulous cases, while HA-coated and commercially pure titanium screw implants were placed in maxillary fully edentulous cases. HA-coated cylinder implants were placed in mandibular partially edentulous cases and in fully edentulous cases in both arches. Any comparisons by implant type must therefore control for the potential confounding effects of the different applications and jaw locations in which the different types of implants were placed.

The relationships between peri-implant vertical bone loss and various patient and treatment-related variables were also investigated. Figure 14 shows bone change by gender. Although differences appeared in some of the study intervals, there seemed to be no clear pattern of bone loss by gender. Study females experienced more bone loss in the intervals between uncovering and the 36-month follow-up. Even less consistent bone loss differences by race/ethnicity are seen in Figure 15. Race/ethnicity may not be a useful predictor of bone loss around placed implants.

Figure 16 shows bone loss by ASA medical status. There were few study patients with ASA medical status of 3. Implants in these patients experienced less bone loss in the early intervals and appeared to have generally experienced more bone loss in the later intervals. Little difference was seen between ASA-1 and ASA-2 status. More bone loss might be expected for patients in worse general health. Selection of patients with compromised general health was left to the dis- cretion of the investigators, however, and investigators may have been more cautious in only selecting patients in which other dental and patient factors besides ASA status were optimal.

Figure 17 displays results for bone loss by smoking status. Although differences were not large, there was a general tendency for more bone loss in smokers. Little difference was seen between former smokers and those who never smoked. Differences were more pronounced in early intervals, which may indicate a stronger effect of smoking in the early stages of healing and stabilization of implants.

The amount of operator experience prior to the DICRG studies was also investigated. Figure 18 shows bone loss results by operator experience. "Less experienced" operators were defined as those placing less than 50 implants prior to the start of the DICRG studies. "More experienced" operators had placed 50 or more implants before the DICRG studies. Most intervals showed slightly less bone loss associated with operators with less prior experience, although the differences were extremely small. Any real difference might again be attributed to patient selection, with "less experienced" operators possibly showing more caution in selecting patients with characteristics optimizing chances for success.

Bone loss results stratified by antibiotic use are shown in Figures 19 and 20. Figure 19 shows no clear pattern of an effect on bone loss with the use of preoperative antibiotics. Figure 20, however, shows a pattern similar to the one seen with HA versus nonHA implants for the use of postoperative antibiotics. In the stages up to the 6-month follow-up, the use of postoperative antibiotics was actually associated with greater bone loss. After the 6-month follow-up, those patients treated with postoperative antibiotics consistently showed less bone loss through the remaining study intervals. Factors affecting the decision to use antibiotics and biologic mechanisms associated with antibiotic usage must be studied more carefully. The decision to use antibiotics might certainly be associated with more questionable patients and treatment conditions. Furthermore, the biologic effects of antibiotics over time after implant insertion must be better understood. Unless the use of antibiotics is totally randomized, other factors correlated with antibiotic usage must be assumed to have a role in these results.

The results presented in this paper provide an overview of some of the factors that may be associated with peri-implant vertical bone loss. More complex analysis must now be conducted to determine which factors are most predictive of bone loss. Some of the possible correlated and confounding relationships among variables with the outcome of vertical peri-implant bone loss have been discussed. Other characteristics of the DICRG radiographic bone loss 
data sets also influence the selection of methods for subsequent analysis. The primary outcome of interest in the DICRG studies is success (or failure) of the placed prostheses and, secondarily, success of the implants supporting the prostheses, which are dichotomous outcomes. The assessment of vertical bone loss, however, involves continuous measurement data and requires different analysis methods. Additionally, the longitudinal analysis (repeated measures) and the clustering of placed implants within patients and study cases require methods to account for correlation in the data.

Many methods for analysis of this type of dental data have been described and used. ${ }^{58-61}$ Preparation of the DICRG radiographic bone loss data set for mixed modeling analysis is in progress. Mixed modeling methods can be used for longitudinal data with a correlated structure and assess fixed and random effects on a continuous outcome; in this case, the extent of vertical bone loss. ${ }^{62-64}$ The results presented in this paper will be used for developing these mixed models. Model development should ideally involve variable selection based on plausible causal mechanisms and relationships of predictor variables to the outcome of interest. The results presented in this paper will be used for variable selection. With objective variable selection, models will be able to assess the effects of the different predictor variables controlling for the effects of other variables included in the model. The real effects of each variable thus may be more validly assessed.

Carrying over into the further analysis will be the dominant trend of dramatically decreasing rate of bone loss over time after implant placement. This decreasing rate of bone loss was seen over all levels of all variables. Most peri-implant bone loss occurs in the first year after implant placement, followed by a small continuing loss of bone. The differences lie in the amount of early bone loss and the amount of decrease in bone loss rate over time. Factors that appear to be associated with early bone loss and with bone loss after this apparent stabilizing of bone levels must be further investigated. Further research should also focus on the implants at the extremes of the distributions. Both those implants which have rapid and continuing bone loss, and those which lose very little or possibly even gain bone, should be investigated specifically. The determination of factors associated with the successful and unsuccessful implants, and factors associated with peri-implant bone loss in general, will contribute to successful future dental implant treatment and improved patient care.

\section{Human Subject Clearance}

All human subject clearance requirements for this research have been completed as part of the main
DICRG studies. The investigation described in this proposal does not expand beyond the main studies in any areas requiring further human subject review or clearance procedures. Proper informed consent has been obtained from all subjects participating in the studies.

\section{REFERENCES}

1. Weiss CM. Short- and long-term bone maintenance surrounding fibro-osteal and osteal integrated dental implants. J Oral Implantol 1990;16:12-19.

2. Brånemark P-I. Introduction to osseointegration. In: Brånemark P-I, Zarb GA, Albrektsson T, eds. TissueIntegrated Prostheses: Osseointegration in Clinical Dentistry. Chicago: Quintessence; 1985:11-76.

3. Lake FT. Basic bone biology in implantology. In: McKinney RV, ed. Endosteal Dental Implants. St. Louis: Mosby Year Book; 1991:52-62.

4. Tatum OH, Lebowitz MS. Anatomic considerations for dental implants. J Oral Implantol 1991;17:16-21.

5. Fagan MJ. New Concepts in Implant Dentistry "Implantodontics," 4th ed. Atlanta: Dental Practice Plan; 1972: Chapters III, V, VII.

6. Chiarenza AR. Retrospective observations on the influence of bone type in determining the nature of bone implant interface. Int J Oral Implantol 1989;6:43-48.

7. Widera GEO, Tesk JA, Privitzer E. Interaction effects among cortical bone, cancellous bone, and periodontal membrane of natural teeth and implants. J Biomed Mater Res 1976;10:613-623.

8. Boyne PJ, Rothstein SS, Gumaer KI, Drobeck HP. Longterm study of hydroxylapatite implants in canine alveolar bone. J Oral Maxillofac Surg 1984;42:589-594.

9. Meffert RM, Block MS, Kent JN. What is osseointegration? Int J Periodontics Restorative Dent 1987;7(4):9-21.

10. Baier RE, Mayer AE. Future directions in surface preparation of dental implants. J Dent Educ 1988;52:788-791.

11. Smith DC. Future directions for research on materials and design of dental implants. J Dent Educ 1988;52:815820.

12. Weiss CM. Dental implants-Differential diagnosis and treatment planning. NY State Dent $J$ 1981;47:12-18.

13. Scheer P, Boyne PJ. Maintenance of alveolar bone through implantation of bone graft substitutes in tooth extraction sockets. J Am Dent Assoc 1987;114:594-597.

14. Albrektsson T, Zarb G, Worthington P, Eriksson AR. The long-term efficacy of currently used dental implants: A review and proposed criteria of success. Int J Oral Maxillofac Implants 1986;1:11-25.

15. Schnitman PA, Shulman LB. Recommendations of the consensus development conference on dental implants. J Am Dent Assoc 1979;98:373-377.

16. Kapur KK. Veterans Administration Cooperative Dental Implant Study-Comparisons between fixed partial dentures supported by blade-vent implants and removable partial dentures. Part II: Comparisons of success rates and periodontal health between two treatment modalities. J Prosthet Dent 1989;62:685-703.

17. Weber HP, Buser D, Fiorellini JP, Williams RC. Radiographic evaluation of crestal bone levels adjacent to nonsubmerged titanium implants. Clin Oral Implants Res 1992;3:181-188.

18. Brånemark P-I, Adell R, Albrektsson T, Lekholm U, Lundkvist S, Rockler B. Osseointegrated titanium fixtures in the treatment of edentulousness. Biomaterials 1983;4:25-28.

19. Nishimura K, Itoh T, Takaki K, Hosokawa R, Naito T, Yokota M. Periodontal parameters of osseointegrated 
dental implants. A 4-year controlled follow-up study. Clin Oral Implants Res 1997;8:272-278.

20. Malmqvist JP, Sennerby L. Clinical report on the success of 47 consecutively placed Core-Vent implants followed from 3 months to 4 years. Int J Oral Maxillofac Implants 1990;5:53-60.

21. Arvidson K, Bystedt H, Frykholm A, von Konow L, Lothigius E. Five-year prospective follow-up report of the Astra Tech dental implant system in the treatment of edentulous mandibles. Clin Oral Implants Res 1998; 9:225-234.

22. Lindquist LW, Carlsson GE, Jemt T. A prospective 15year follow-up study of mandibular fixed prostheses supported by osseointegrated implants. Clin Oral Implants Res 1996; 7:329-336.

23. Pilliar RM, Deporter PA, Watson PA, Valiquette N. Dental implant design-Effect on bone remodeling. J Biomed Mater Res 1991;25:467-483.

24. Karlsson U, Gotfredsen K, Olsson C. A 2-year report on maxillary and mandibular fixed partial dentures supported by Astra Tech dental implants. Clin Oral Implants Res 1998;9:235-242.

25. Teixeira ER, Sato Y, Akagawa Y, Kimoto T. Correlation between mucosal inflammation and marginal bone loss around hydroxyapatite-coated implants: A 3-year crosssectional study. Int J Oral Maxillofac Implants 1997;12: 74-81.

26. Jepsen S, Tuhling A, Jepsen K, Ohlenbusch B, Albers HK. Progressive peri-implantitis. Incidence and prediction of peri-implant attachment loss. Clin Oral Implants Res 1996; 7:133-142.

27. Isidor F. Loss of osseointegration caused by occlusal load of oral implants. A clinical and radiographic study in monkeys. Clin Oral Implants Res 1996;7:143-152.

28. Lindquist LW, Carlsson GE, Jemt T. Association between marginal bone loss around osseointegrated mandibular implants and smoking habits: A 10-year follow-up study. $J$ Dent Res 1997;76:1667-1674.

29. Malevez CH, Hermans M, Daelemans PH. Marginal bone levels at Brånemark system implants used for single tooth restoration: The influence of implant design and anatomical region. Clin Oral Implants Res 1996;7:162169.

30. De Bruyn H, Collaert B, Linden U, Johansson C, Albrektsson T. Clinical outcome of Screw Vent implants. A 7-year prospective follow-up study. Clin Oral Implants Res 1999;10:139-148.

31. Cochran DL, Nummikoski PV, Higginbottom FL, Hermann HS, Makins SR, Buser D. Evaluation of an endosseous titanium implant with a sandblasted and acid-etched surface in the canine mandible: Radiographic results. Clin Oral Implants Res 1996;7:240-252.

32. Norton MR. Marginal bone levels at single tooth implants with a conical fixture design. The influence of surface macro- and microstructure. Clin Oral Implants Res 1998;9:91-99.

33. Bragger U, Hafeli U, Huber B, Hammerle CHF, Lang NP. Evaluation of postsurgical crestal bone levels adjacent to non-submerged dental implants. Clin Oral Implants Res 1998;9:218-224.

34. Pham AN, Fiorellini JP, Paquette D, Williams RC, Weber HP. Longitudinal radiographic study of crestal bone levels adjacent to non-submerged dental implants. J Oral Implantol 1994;20:26-34.

35. Schei O, Waerhaug J, Lovdal A, Arno A. Alveolar bone loss as related to oral hygiene and age. J Periodontol 1959;30:7-16.
36. Bjorn H, Holmberg K. Radiographic determination of periodontal bone destruction in epidemiological research. Odont Revy 1966;17:232-250.

37. Bjorn AL. Dental health in relation to age and dental care. Odont Revy 1974;25(Suppl. 29):8-13.

38. Douglass CW, Valachovic RW, Wijesinha A, Chauncey $\mathrm{HH}$, Kapur KK, McNeil BJ. Clinical efficacy of dental radiography in the detection of dental caries and periodontal diseases. Oral Surg Oral Med Oral Pathol 1986;62:330-339.

39. Akesson L, Hakansson J, Rohlin M. Comparison of panoramic and intraoral radiography and pocket probing for the measurement of the marginal bone level. $J$ Clin Periodontol 1992;19:326-332.

40. Gettleman L, Schnitman PA, Kalis P, et al. Clinical evaluation criteria of tooth implant success. J Oral Implantol 1978;8:12-28.

41. Grondahl K, Sunden S, Grondahl HG. Inter- and intraobserver variability in radiographic bone level assessment at Brånemark fixtures. Clin Oral Implants Res 1998; 9:243-250.

42. Hausmann E, Allen K, Christersson L, Genco R. Effect of $\mathrm{x}$-ray beam vertical angulation on radiographic alveolar crest level measurement. J Periodont Res 1989;24:819.

43. Stenstrom B, Julin P, Lavstedt S. Comparison between panoramic radiographic techniques. Part II: Marginal bone level interpretability with Status-X and Orthopantomograph, model OP 3. Dentomaxillofac Radiol 1982; $11: 37-45$

44. Benn DK. A review of the reliability of radiographic measurements in estimating alveolar bone changes. J Clin Periodontol 1990;17:14-21.

45. Dunn SM, Kantor ML. Digital radiology facts and fictions. J Am Dent Assoc 1993;124:39-47.

46. van der Stelt PF. Modern radiographic methods in the diagnosis of periodontal disease. Adv Dent Res 1993; 7:158-162.

47. Bragger U. Digital imaging in periodontal radiography. $J$ Clin Periodontol 1988;15:551-557.

48. Jeffcoat MK. Radiographic methods for the detection of progressive alveolar bone loss. J Periodontol 1992;63: 367-372.

49. Jeffcoat MK, Reddy MS. Digital subtraction radiography for longitudinal assessment of peri-implant bone change: Method and validation. Adv Dent Res 1993;7: 196-201.

50. Bragger U, Litch J, Pasquali L, Kornman KS. Computer assisted densitometric image analysis for the quantitation of radiographic alveolar bone changes. J Periodont Res 1987;22:227-229.

51. Bragger U, Pasquali L, Kornman KS. Remodeling of interdental alveolar bone after periodontal flap procedures assessed by means of computer-assisted densitometric image analysis (CADIA). J Clin Periodontol 1988;15:558-564.

52. Benn DK. A computer-assisted method for making linear radiographic measurements using stored regions of interest. J Clin Periodontol 1992;19:441-448.

53. Hausmann E, Dunford R, Wikesjö U, Christersson L, McHenry K. Progression of untreated periodontitis as assessed by subtraction radiography. $J$ Periodont Res 1986;21:716-721.

54. American Society of Anesthesiologists. New classification of physical status. Anesthesiology 1963;24:111.

55. Lambert PM, Morris HF, Ochi S. Positive effect of surgical experience with implants on second-stage implant 
survival. J Oral Maxillofac Surg 1997;55(Suppl. 5):1218.

56. Misch CE. Progressive loading of bone with implant prostheses. J Dent Sympos 1993;1:50-53.

57. Orenstein IH, Synan WJ, Truhlar RS, Morris HF, Ochi S. Bone quality in patients receiving endosseous dental implants: DICRG interim report no. 1. Implant Dent 1994;3:90-94.

58. Albandar JM, Rise J, Gjermo P, Johansen JR. Radiographic quantification of alveolar bone level changes. A 2-year longitudinal study in man. $J$ Clin Periodontol 1986;13:195-200.

59. Albandar JM. Some predictors of radiographic alveolar bone height reduction over 6 years. J Periodont Res 1990;25:186-192.

60. Wennstrom JL. Interpretation of radiographic data on longitudinal loss of periodontal support. J Periodontol 1990;61:459-460.

61. Wallenstein S, Fleiss JL, Kingman A. Repeat measurements analysis of dental data. J Dent Res 1980;59:2021 2024.
62. Verbeke G, Molenberghs G. Linear Mixed Models in Practice. New York: Springer Verlag; 1997:1-30, 63-155.

63. Littell RC, Milliken GA, Stroup WW, Wolfinger RD. SAS System for Mixed Models. Cary, NC:SAS Institute; 1996: 87-134.

64. Latour D, Littell R. Advanced General Linear Models With an Emphasis on Mixed Models. Cary, NC:SAS Institute; 1996:38-43, 269-416.

Accepted for publication October 13, 2000. 